\title{
Experimental Investigation of the Rail Pressure Fluctuations Correlated with Fuel Properties and Injection Settings
}

\author{
Carlo Coratella ${ }^{1}$. Lewis Parry ${ }^{1}$ Yanfei $\mathrm{Li}^{2} \cdot$ Hongming $\mathrm{Xu}^{1,2}$
}

Received: 5 March 2020 / Accepted: 31 December 2020 / Published online: 23 April 2021

(c) The Author(s) 2021

\begin{abstract}
Injection-induced rail pressure fluctuations are proven to cause nonuniform spray development. These fluctuations are also responsible for generating lower injection pressures, to the detriment of jet penetration length and break-up timing. Despite the vast literature dealing with such issues, several aspects of rail pressure fluctuations remain unclear. Additionally, the need for compliance with the emission legislation has shed light on the potential of alternative fuels, which represent a pathway for sustainable mobility. This scenario has motivated the present study dealing with the assessment of the time history of rail pressure correlated with fuel properties. Tests have been performed using a last-generation common rail injection equipment under various injection settings, employing diesel and 2-methylfuran-diesel blend. This paper describes the research activity and aims to provide new insights into the correlation of rail pressure fluctuations with fuel properties.
\end{abstract}

Keywords Rail pressure fluctuations $\cdot$ Wave propagation $\cdot$ Viscosity $\cdot$ Bulk modulus $\cdot 2$-Methylfuran $\cdot$ Fuel blend

\section{Introduction}

The massive introduction of the electronically managed injection system is mainly due to the flexibility and fast response of the injection system components. However, additional refinements on modern engines have been demanded by the emission legislation over the last decades. This has led the engine manufacturers to design the MultiJet system, which assures considerably faster injector actuation, enabling the injection cycle to be split up into eight separate shots. Such a technology has been demonstrated to appreciably improve the accuracy of the injected fuel amount. On the other hand, the fast dynamics of common rail (CR) hydraulic components engender sensible pressure perturbations, which leads to spray nonuniformity, adversely affecting the combustion efficiency.

Hongming Xu

h.m.xu@bham.ac.uk

1 School of Mechanical Engineering, University of Birmingham, Edgbaston, Birmingham B15 2TT, UK

2 State Key Laboratory of Automotive Safety and Energy, Tsinghua University, Beijing, China

\subsection{Pressure Fluctuations in the Modern Injection System}

Once the injection commences, the upward needle motion gives rise to a rarefaction wave traveling from the injector nozzle towards the rail $[1,2]$. As the pressure wave reaches the common rail, pressure fluctuations occur. In this regard, a vast literature demonstrated that injection-induced pressure fluctuations lead to the shot-to-shot variability underlying the deviations from the expected mass flow rate. Additionally, it is well known that the stroke-by-stroke motion of the pump significantly contributes to rail pressure fluctuations. This framework has driven the research community to develop several predictive models and devices to control or even compensate for the rail pressure oscillations.

Ubertini et al. [3] have developed a multidimensional code to simulate pressure pulsations taking place upstream of the injector nozzle. More specifically, numerical results on a 3D virtual spray injected into a constant pressure vessel have revealed the significant role of injection pressure fluctuations in the injected flow rate and spray characteristics. It emerged also that higher injection pressures lead to larger fluctuations amplitude.

Likewise, Beierer et al. [4] designed a numerical model of a commercial CR system, developed in the GT-FUEL (Gamma Technologies, Inc.) software and aimed to predict 
pressure oscillations at the injector inlet. The investigation has demonstrated that pressure fluctuations are meaningfully determined by injection pressure, injection duration, and connection pipe geometry. It has emerged that the minimization of pressure variation is achievable via shorter injection duration combined with lower injection pressure. Further mitigation is obtainable via a large connection line diameter and a shorter connection line.

Catalano et al. [5] developed a CFD code for simulating the unsteady flow in the pipes of a common-rail system for diesel engines. The main finding consists of the demonstration that the possible reduction in injection pressure over a split injection cycle is mainly ascribable to dynamical effects rather than to friction losses. This may discourage all attempts to minimize the friction losses, which are commonly deemed responsible for a possible decrease in injection pressure.

A thorough insight into the cause-and-effect relationship between the injection-induced pressure oscillation and the system hydraulic layout has been provided by Catania et al. [6], who designed a zero-dimensional mathematical model allowing the investigation of the highly unsteady wave propagation occurring in the $\mathrm{CR}$ system. Paying attention to the multi-injection process, the model has revealed that the impact of injection-induced pressure fluctuations on the injected volume is linked to dwell time between consecutive injections.

Further insight has been provided by Pontoppidan et al. [7], whose research dealt with the role of pressure fluctuations at the injector inlet in the mixture preparation and spray stability. The investigation has revealed that pressure oscillations upstream of the nozzle hinder the spray stability. On the other hand, it has been observed that the influence of pressure fluctuations on spray stability is weakened with an increasing number of split injections.

Along with the above research activities, the scientific community has designed and built several devices devoted to regulating or even suppressing the fuel pressure pulsation occurring at the injector inlet and within the rail $[8,9]$.

\subsection{State of Art of 2-Methylfuran-Based Fuel Blends}

Alternative fuels have been proven to substantially reduce tailpipe emissions, providing a trade-off solution combining the engine efficiency with compliance with environmental mandates. Bio-ethanol is widely employed, owing to its renewable nature and high octane number. However, the large volatility, low energy density, and high energy consumption in the production process discourage a wider spread.

This framework has driven the researchers to shift the attention to furan-based fuels, the production of which is feasible via a methodology designed by Dumesic and Román
[10]. This method represents a noticeable breakthrough and has been improved by Zhao et al. [11], whose approach has led to a steep reduction in production costs. Along with the efficient production method, the high renewability of fructose may encourage the commercial aspects of MF-based blends.

Moreover, $\mathrm{HC}$ emissions from 2-methylfuran have been proved to be at least $61 \%$ lower than gasoline while the PM emissions are lower than those of gasoline in DI mode because of the molecular oxygen content.

However, due to the high adiabatic flame temperature of 2-methylfuran, the NOx emission level is still a concern. In this respect, Wang et al. [12] carried out an experimental investigation revealing the impact of low-level 2-methylfuran content in gasoline on the direct-injection spark-ignition (DISI) engine. Particularly, a trade-off between tailpipe emissions and engine efficiency is feasible via a volumetric $10 \%$ of 2-methylfuran content. Moreover, the burning rate is noticeably faster than the gasoline one, owing to the larger vaporization rate. This leads to the presumption that a greater vaporization rate may allow the issues associated with cold engine start being overcome. This physiochemical property may encourage boosted induction technologies and higher SI engines compression ratio, to maximize the thermal efficiency and power.

To summarize, along with the aforementioned production technique, these physicochemical peculiarities make furan blends a promising candidate to supersede the conventional fuels, presenting a potential pathway to supply renewable sources for sustainable mobility.

\subsection{This Study}

The above framework has motivated the authors to undertake a robust experimental campaign under various injection scenarios employing different fuels, such as diesel and diesel-2-methylfuran blend. The present study aims to provide additional insight into the rail pressure fluctuations, induced by the injection event and correlated with fuel properties. More specifically, along with injection setting, this study identifies viscosity, density, and bulk modulus as the key parameters determining rail pressure fluctuations.

\section{Test Facilities and Experimental Setup}

Figure 1 provides a schematic overview of the bench employed for the tests. The rig is a STPiW2 type, a lastgeneration CR equipment manufactured by Autoelektronika.

The rig includes a high-pressure pump, which can supply injection pressures up to $150 \mathrm{MPa}$. The injection pressure and rotational speed of the electric motor are managed via a dedicated control unit and a single-phase inverter, 


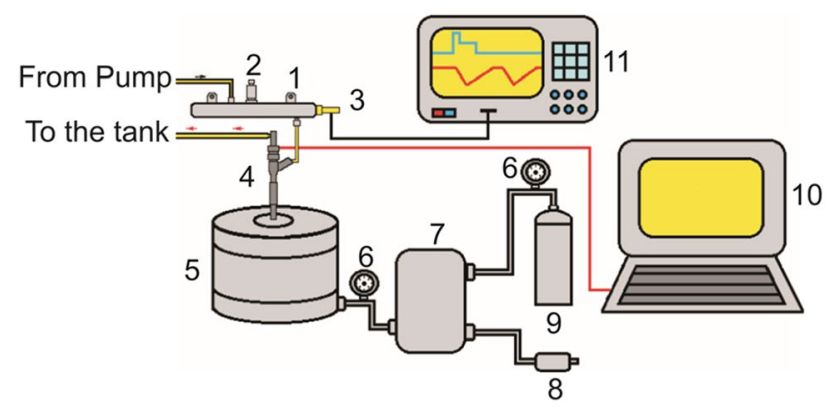

Fig. 1 Test rig: 1 -common rail; 2-pressure regulating valve; $3-$ rail pressure sensor; 4-injector; 5-high-pressure vessel; 6-pressure gauges; 7-pressurized air chamber; 8-relief valve; 9-pressurized air tank; 10 - computer; 11 -oscilloscope

respectively. The return fuel is cooled through a heat exchanger before flowing back towards the tank. A microcontroller displays the fuel temperature at the inlet and outlet of the heat exchanger. The injector is a solenoid-driven CR injector, manufactured by Denso and provided with seven holes. The fuel injector is fitted to a high-pressure vessel, which can store backpressures up to $11 \mathrm{MPa}$.

Injection timing and duration are set via a dedicated program implemented in the ECKA software. A sensor, with an accuracy range of $1.5 \%$ at full scale and a response time of $2 \mathrm{~ms}$, is mounted on the common rail to detect the rail pressure. The pressure sensor is connected to an oscilloscope, which enables the visualization of the time history of the rail pressure.

In order to visualize the current profile driving the injector, the oscilloscope is connected also to the injector solenoid.

\subsection{Calculation of Fuel Blend Properties}

Table 1 provides the diesel and 2-methylfuran properties, which are deemed to determine significantly the rail pressure oscillations.

Bearing in mind that the temperatures detected here range from 30 to $50{ }^{\circ} \mathrm{C}$, the diesel viscosities have been obtained according to the correlation found by Wang et al. [13]. The densities have been obtained on the basis of findings achieved by Esteban et al. [14]. Concerning the 2-methylfuran, Jezak et al. [15] demonstrated that temperature exerts a noticeable impact on the furan density.
The study includes also the calculation of speed of sound across the furan-based fuels at various temperatures. For this study, the approximated estimation of the bulk modulus is achieved via the formula:

$c \approx \sqrt{\frac{K}{\rho}}$

with $c, K$, and $\rho$ being the speed of sound, bulk modulus, and density, respectively. In this regard, it is inferred that a larger bulk modulus promotes wave propagation [16].

The listed densities and viscosities permit the calculation of the overall properties of the furan-based blends. The tested blends are referred to as MF30 and MF50, since they are characterized by a volumetric composition of $30 \%$ and $50 \%$ of 2-methylfuran, respectively. In this regard, an overview of the methods calculating the blends' viscosity and density is needed. However, since this study is devoted to assessing the influence of fuel properties on the rail pressure variation, the ranking of viscosity and density of blends is deemed sufficient for the work.

\subsubsection{Calculation of Fuel Blend Viscosity}

Although the progress lifts the blending viscosity estimation to a satisfactory accuracy, nowadays there is not any universal theory providing the exact calculation. The majority of the existing theories are limited to the ideal binary blends, in which mixing operation does not produce any volume change. Therefore, viscosity prediction for binary mixtures remains a challenging task due to the large degree of the blending curves nonlinearity. However, empirical formulas linking the single component's viscosity to blend overall one have been developed [17].

Table 2 shows the ranges of the blends viscosities, the values of which are achieved via the most employed methods. The viscosities range according to the detected fuel temperature.

\subsubsection{Estimation of Fuel Blend Density}

Similarly to the calculation of blend overall viscosity, there is not any general formula to calculate the overall density of a blend compounded with two miscible liquids. However, the following formula is commonly employed:

$\rho_{\mathrm{TOT}}=\Sigma \chi_{\mathrm{i}} \rho_{\mathrm{i}}$
Table 1 The properties of the fuels constituting the blends

\begin{tabular}{llcl}
\hline & Kinematic viscosity $\left(\mathrm{m}^{2} / \mathrm{s}\right)$ & Density $\left(\mathrm{kg} / \mathrm{m}^{3}\right)$ & Bulk modulus $(\mathrm{MPa})$ \\
\hline 2-Methylfuran & $4.31 \times 10^{-6}$ & $880-915$ & $1958.41-2071.65$ \\
Diesel & $8.70 \times 10^{-7}-2.50 \times 10^{-6}$ & $807.97-830.7$ & 1545 \\
\hline
\end{tabular}


Table 2 The ranges of blends viscosities obtained by different approaches

\begin{tabular}{lrll}
\hline & Viscosity by Refutas $\left(\mathrm{m}^{2} / \mathrm{s}\right)$ & $\begin{array}{l}\text { Viscosity by Kendall- } \\
\text { Monroe }\left(\mathrm{m}^{2} / \mathrm{s}\right)\end{array}$ & Viscosity by Arrhenius $\left(\mathrm{m}^{2} / \mathrm{s}\right)$ \\
\hline MF30 & $1.32 \times 10^{-6}-3.86 \times 10^{-6}$ & $1.59 \times 10^{-6}-3.87 \times 10^{-6}$ & $1.46 \times 10^{-6}-3.86 \times 10^{-6}$ \\
MF50 & $1.76 \times 10^{-6}-3.98 \times 10^{-6}$ & $2.19 \times 10^{-6}-3.99 \times 10^{-6}$ & $1.99 \times 10^{-6}-3.98 \times 10^{-6}$ \\
\hline
\end{tabular}

where $\chi_{\mathrm{i}}$ and $\rho_{\mathrm{i}}$ refer to weight fraction and density of the $i$ th component, respectively.

Accordingly, the MF30 density ranges from 827.60 to $850.14 \mathrm{~kg} / \mathrm{m}^{3}$, while the MF50 one varies from 840.69 to $866.18 \mathrm{~kg} / \mathrm{m}^{3}$.

\section{Results and Discussion}

\subsection{Experimental Tests}

A comprehensive investigation has been conducted, so as to assess the rail pressure fluctuations. As mentioned above, the blends are referred to as MF30 and MF50. The injection pressure was set to $40,70,100$, and $140 \mathrm{MPa}$, while the backpressure was set to $2,4,6$, and $8 \mathrm{MPa}$, for a total of 16 injection configurations. The energizing time was set to $2000 \mu$ s. Figure 2 depicts the time history of rail pressure and current profile related to a series of four injections at backpressure of $2 \mathrm{MPa}$. For the sake of brevity, the charts related to the rest of the backpressures are omitted.

As expected, higher injection pressures imply greater pressure drop, which has been revealed to vary also with the fuel characteristics. The curves reveal also that once the injection commences, fuel pressure is first subjected to a steep drop, followed by an increase until it stabilizes around the pressure value preceding the injection process. Furthermore, the interval between the timings corresponding to the lowest pressure and the one preceding the injection start is observed to vary both with injection settings and with fuel properties. From the graphs, it emerges also that a discrepancy between the set energizing time and current duration of the injector opening stage occurs. This phenomenon may be attributable to the on-board capacitors on the injection control unit that are charged with current over a set period before discharging at a higher current for a shorter period. Section 3.2 describes the above findings in detail.

\subsection{Analysis of the Experimental Findings}

The following paragraphs provide an accurate explanation of the experimental findings, singling out the key characteristics describing the dynamical effects in the rail as below:

1. The pressure drop stemming from needle lift;
2. The retrieving time, namely the interval between timings corresponding to the lowest pressure and the one preceding the following injection start;

3. The amplitude of pressure fluctuations;

4. The time between two consecutive pressure peaks during the increasing stage of rail pressure;

\subsubsection{Rail Pressure Drops}

From the above curves, it emerges that higher injection pressures give rise to larger pressure drops. In this regard, the here detected variations in pressure differ from the ones that arose in the injector nozzle. Figure 3 illustrates the sectional view of a diesel injector.

Once the injector solenoid (Component 1 ) is energized, the valve ball (Component 2) is dragged up and moves upward, allowing the fuel to flow through the Abfluss hole (Component 3). Hence, a dramatic reduction in pressure occurs within the control chamber (Component 4). This results in the upward motion of the assembly made up of the needle (Component 5) and control piston (Component 6), thanks also to fuel pressure acting on the needle protrusion. As a consequence, the injection process commences. However, the high-pressure pump continues to supply fuel, which is delivered to the control chamber. As a result, the above reduction in control chamber volume gives rise to a compression wave, which is conveyed to the rail. Additionally, the steep reduction in fuel pressure, caused by the needle lift, generates a rarefaction wave propagating toward the rail. Due to the difference in path lengths, the aforementioned compression wave precedes the rarefaction one [18]. Moreover, it should be kept in mind that once the rarefaction wave reaches a changing section, it gives rise to a reflecting compression wave which travels back to the delivery chamber. To summarize, these wave phenomena are the main cause for the difference between the pressure variation in the nozzle and the one taking place in the rail. Figure 4 illustrates the averages of pressure drops detected at each injection shot. The diagrams enable the comparison between the pressure drops regarding each fuel, so as to remark the dependence of variation in rail pressure on the fuel characteristics. Since the trends of rail pressure have been observed to do not vary considerably with backpressure, only figures referring to backpressure 2 and $8 \mathrm{MPa}$ are shown.

As expected, along with higher viscosity, a larger density is revealed to alleviate the pressure drop induced by the 


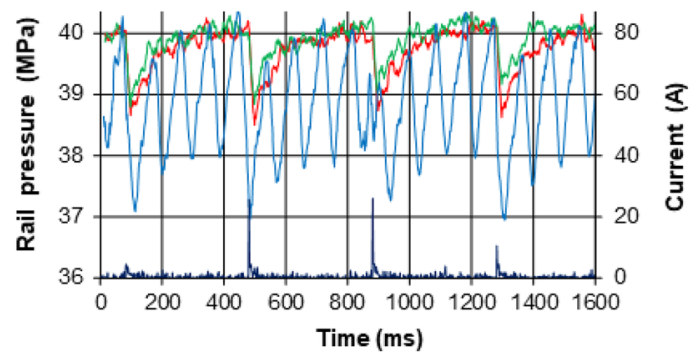

(a)

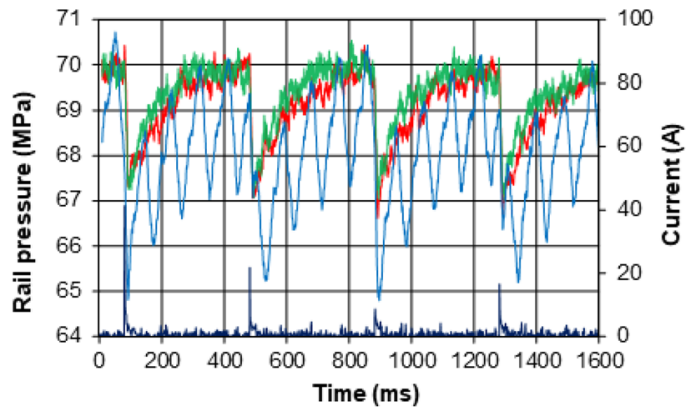

(b)

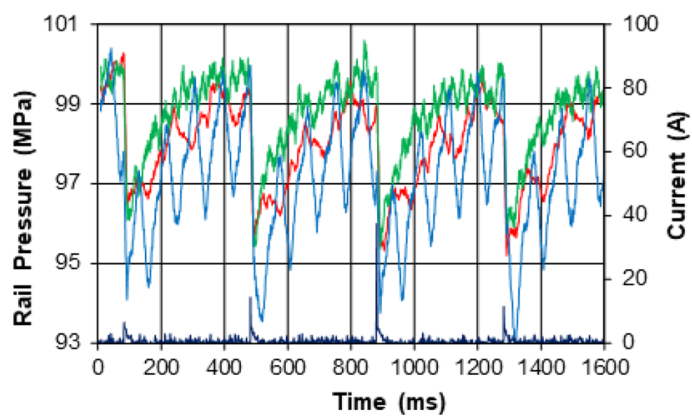

(c)

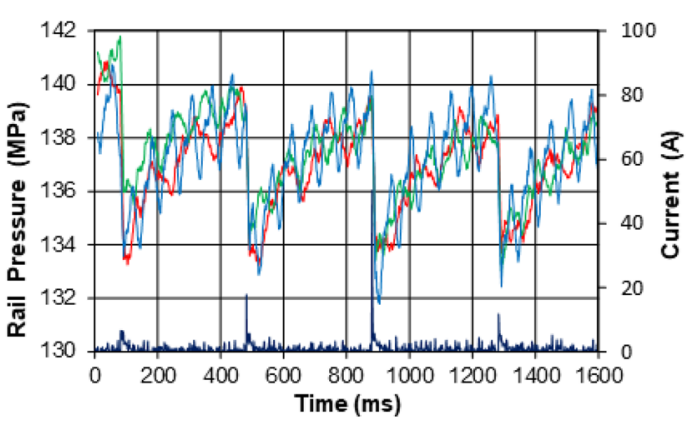

(d)

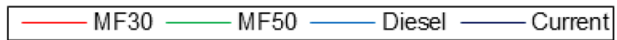

Fig. 2 Time history of rail pressure at backpressure of $2 \mathrm{MPa}$ and a injection pressure $40 \mathrm{MPa}$; b injection pressure $70 \mathrm{MPa}$; $\mathbf{c}$ injection pressure $100 \mathrm{MPa}$; d injection pressure $140 \mathrm{MPa}$

commencing injection. The above findings can be explained by Bernoulli's equation, which describes the fluid flow.

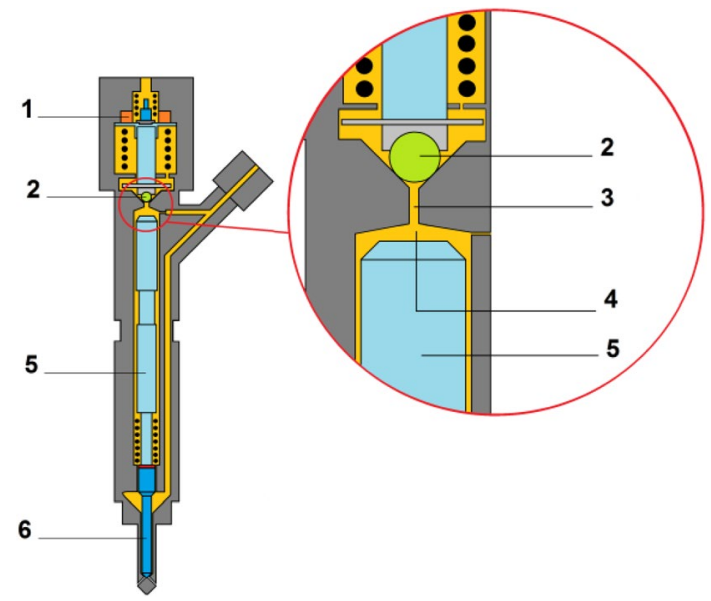

Fig. 3 Sectional view of common rail injector

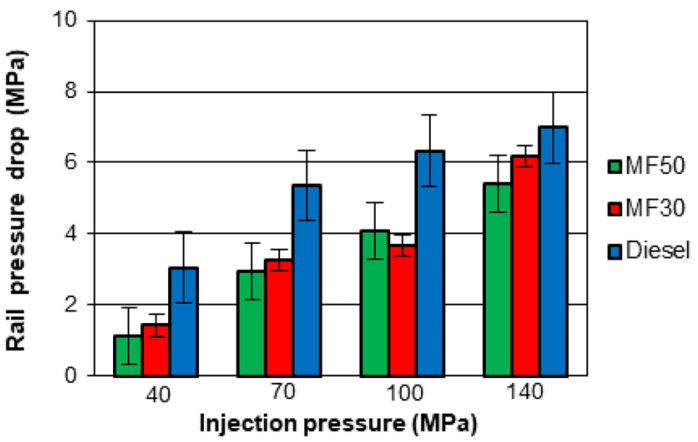

(a)

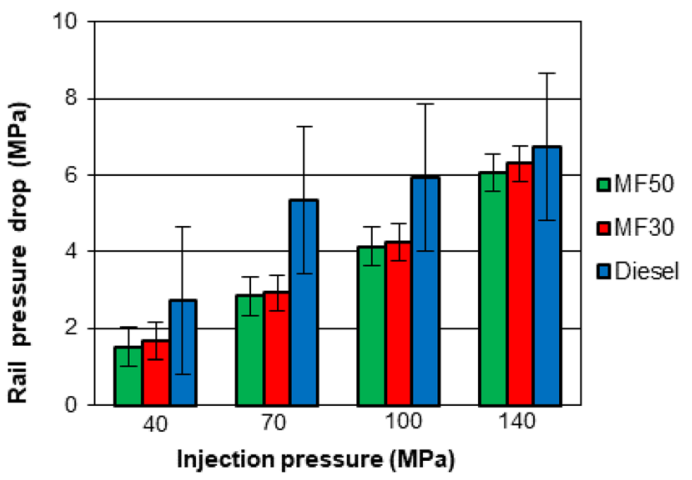

(b)

Fig. 4 Averaged reductions in rail pressure, arisen after the injection start, at various injection pressures and a backpressure $2 \mathrm{MPa}$; $\mathbf{b}$ backpressure $8 \mathrm{MPa}$

Greater injection pressure boosts fluid velocity, according to the formula: 
$v \approx \sqrt{\frac{2\left(P_{\mathrm{inj}}-P_{\mathrm{b}}\right)}{\rho_{\mathrm{FUEL}}}}$

where $v, P_{\text {inj }}, P_{\mathrm{b}}$, and $\rho_{\mathrm{FUEL}}$ stand for fuel velocity, injection pressure, backpressure, and fuel density, respectively. Accordingly, decreasing density results in greater fuel velocity, which in turn implies larger pressure drops. Additionally, it is well known that, on an equal injection setting, lower viscosity promotes the velocity of the fluid flow [19]. This is mainly due to the minimization of the boundary layer, leading to the accentuation of the velocity gradient.

As a consequence, greater fluid velocity gives rise to a larger pressure drop in the nozzle orifices. By contrast, larger viscosity accentuates the boundary layer arisen at the orifice walls, at the detriment of the velocity gradient. Therefore, MF50 fuel flow is characterized by the accentuation of the energy dissipation, which results in the slowest motion as well as a lower pressure drop in comparison with diesel and MF30. Additionally, from the experimental campaign, it emerged that, along with injection pressure, backpressure also plays a pivotal role in determining the pressure drops induced by the injection shot. An accurate explanation is provided in Sect. 4.

\subsubsection{Characteristics of Rail Pressure Fluctuations}

As already mentioned, the pressure drop, stemming from the needle lift at the start of injection, generates a rarefaction wave, which reaches the delivery chamber and travels to the rail [6]. The graphs in Fig. 2 evidence also that once the injection occurs, rail pressure experiences an initial dramatic drop followed by an increasing trend until it approaches the value preceding the injection start. This makes rail pressure subjected to dynamical effects, characteristics of which are linked with fuel properties and injection settings. These fluctuations engender overpressures frequently exceeding the set rail pressure. However, the nonstationary trend typically characterizing diesel pressure pulsations hinders the prediction of the injection timing corresponding to overpressure, to the disadvantage of the injection strategy optimization. Figure 5 illustrates the bar charts referring to the averages of the retrieving times, the duration of which is measured between the timings corresponding to the lowest pressure and the pressure preceding the injection start. Since the trend of retrieving time has been revealed to do not vary significantly with backpressure, only figures referring to backpressure 2 and $8 \mathrm{MPa}$ are shown.

It emerges that the duration of such time interval varies according to fuel characteristics. Particularly, the rail pressure of MF50, which is characterized by the largest bulk modulus, requires a shorter time to retrieve the value preceding the previous start of injection in most cases. Like rail

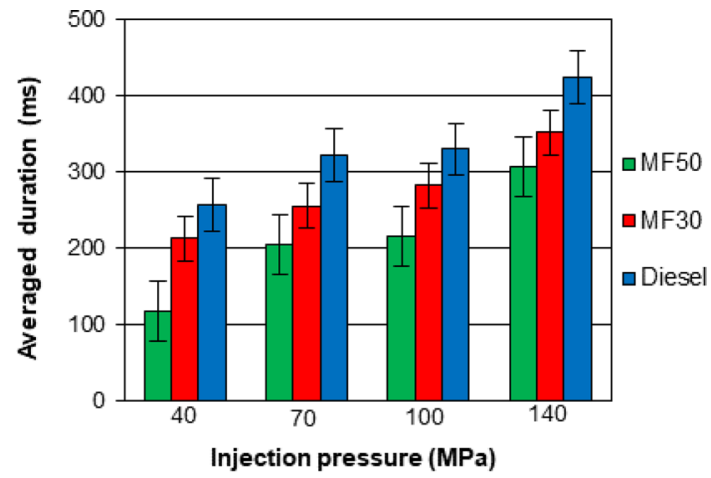

(a)

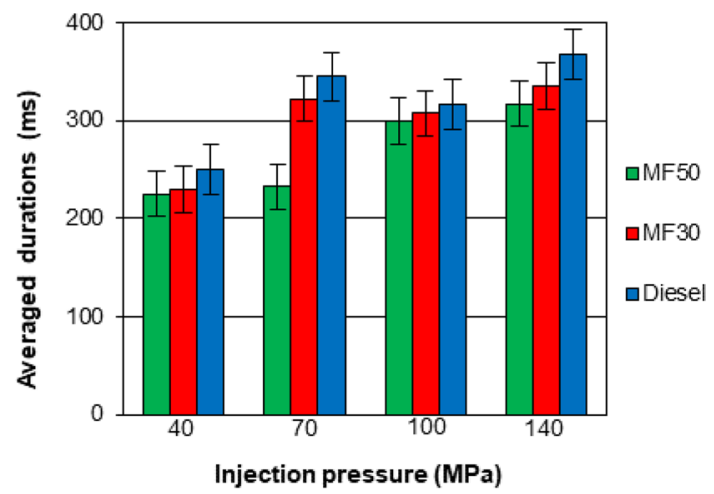

(b)

Fig. 5 Retrieving times at various injection pressures and a backpressure $2 \mathrm{MPa}$; b backpressure $8 \mathrm{MPa}$

pressure drop stemming from the injection, backpressure exerts a non-negligible role on the retrieving time. The reasons underlying this finding are fully described in Sect. 4. Focusing on the time history of rail pressure, it is noteworthy that the increasing trend of the rail pressure is characterized by a series of smaller oscillations, amplitudes of which vary with fuel properties. Figure 6 show the comparison between the averaged amplitudes of pressure fluctuations occurring over the temporal stage during which rail pressure retrieves the value preceding the previous start of injection. In this regard, from the tests, it emerges that the trends of rail pressure do not vary appreciably with backpressure. Therefore, only figures referring to backpressure 2 and $8 \mathrm{MPa}$ are shown.

Similar to what emerging from previous charts, the averaged amplitude is strongly correlated with fuel properties. Likewise, Fig. 7 compare the averaged values of the time interval between two consecutive pressure peaks, indicating the pressure pulsations frequency.

According to Figs. 6 and 7, it emerges that diesel experiences the largest amplitude of rail pressure and the longest time between two consecutive pressure peaks. By contrast, adding 2-methylfuran to the blend composition contributes to the reduction in amplitude as well as to the decrease in 


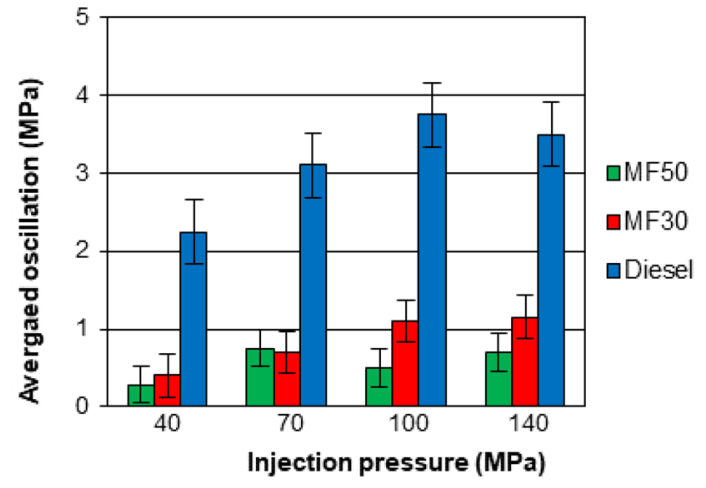

(a)

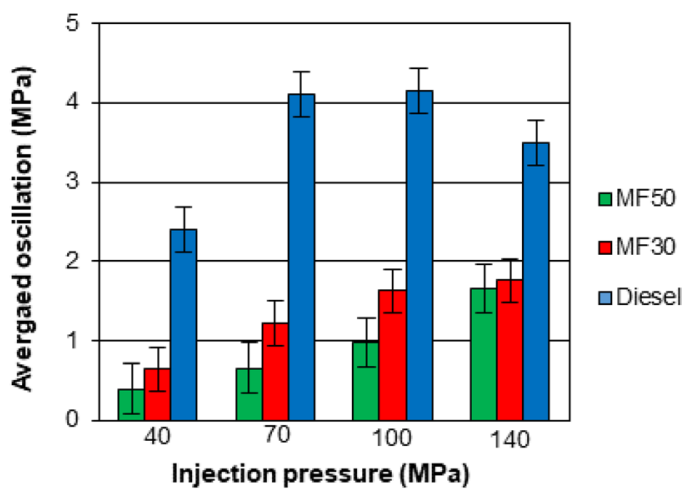

(b)

Fig. 6 Averaged amplitudes of rail pressure fluctuations at various injection pressures and a backpressure $2 \mathrm{MPa}$; $\mathbf{b}$ backpressure $8 \mathrm{MPa}$

the time interval. However, Fig. 7 exhibits some inconsistencies at injection settings of 140-2 MPa, 140-4 MPa, and 140-8 MPa, where the time between two MF30 pressure peaks is longer if compared to diesel. It is reasonable to attribute this inconsistency to the dynamical effects characterizing the diesel pressure fluctuations, which are accentuated by the higher injection pressure. Higher injection pressure is believed to promote pressure instability of diesel, owing to the lower bulk modulus. Hence, this gives rise to unpredictable trends of the diesel pressure within the rail.

\subsubsection{The Influence of the Backpressure on the Rail Pressure Fluctuations}

As abovementioned, along with injection pressure and fuel properties, backpressure exerts a meaningful role in the pressure drops and the retrieving time. The reason underlying this observation is explained in the following paragraphs.

(1) The influence of the backpressure on the rail pressure drops

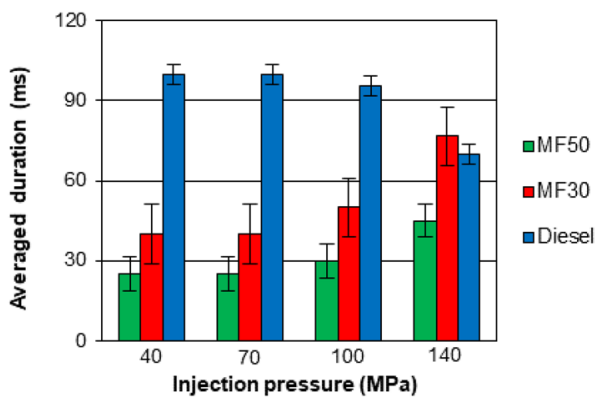

(a)

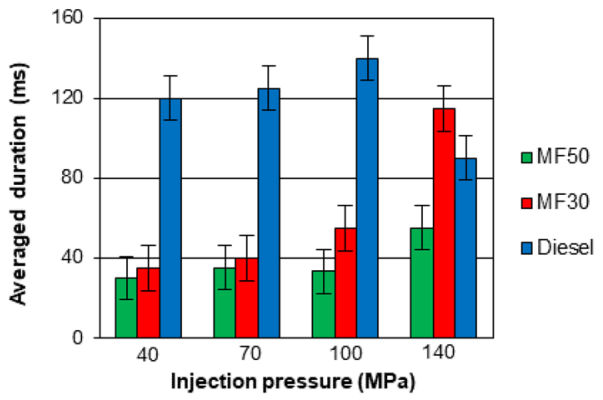

(b)

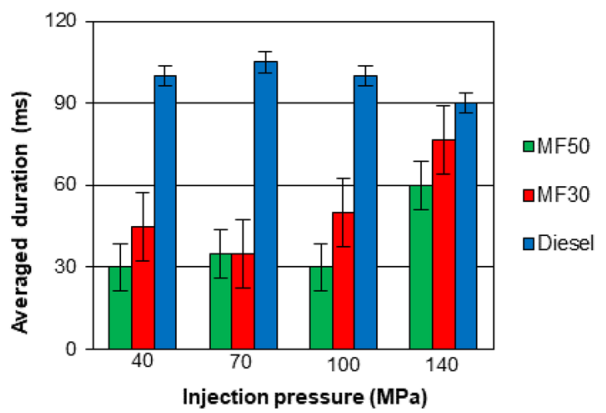

(c)

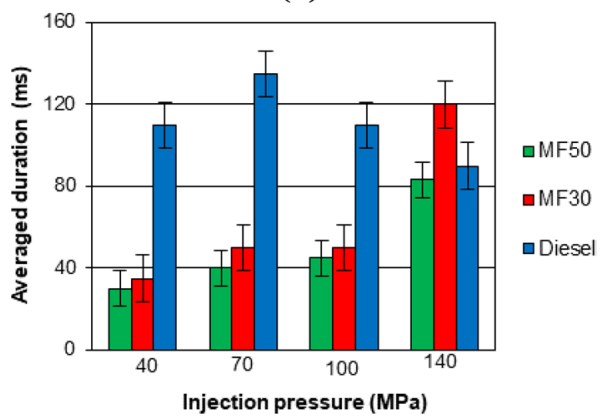

(d)

Fig. 7 Duration between two consecutive pressure peaks while injection pressure stabilises, at various injection pressures and backpressures: a $2 \mathrm{MPa}$; b $4 \mathrm{MPa}$; $6 \mathrm{MPa}$; d $8 \mathrm{MPa}$

Table 3 shows the averaged pressure drops over four injections and referring to Fig. 4.

As expected, diesel exhibits an increasing trend of the pressure drop as a result of the increase in injection pressure. Likewise, both MF50 and MF30 reveal an increasing trend at higher injection pressure and constant backpressure. 
Table 3 Rail pressure drops induced by altering fuels, injection pressure, and backpressure

\begin{tabular}{llllll}
\hline Fuel & $\begin{array}{l}\text { Backpres- } \\
\text { sure (MPa) }\end{array}$ & \multicolumn{4}{l}{ Injection pressure } \\
\cline { 3 - 6 } & $40 \mathrm{MPa}$ & $70 \mathrm{MPa}$ & $100 \mathrm{MPa}$ & $140 \mathrm{MPa}$ \\
\hline \multicolumn{4}{l}{ Rail pressure drop after injection } & $(\mathrm{MPa})$ \\
Diesel & 2 & 3.06 & 5.36 & 6.34 & 6.98 \\
& 4 & 3.14 & 4.42 & 6.26 & 6.96 \\
& 6 & 2.90 & 4.82 & 6.28 & 7.18 \\
& 8 & 2.74 & 5.36 & 5.94 & 6.74 \\
MF30 & 2 & 1.42 & 3.28 & 3.68 & 6.18 \\
& 4 & 1.72 & 3.14 & 4.50 & 6.14 \\
& 6 & 1.68 & 2.94 & 4.66 & 5.60 \\
& 8 & 1.68 & 2.92 & 4.24 & 6.30 \\
MF50 & 2 & 1.14 & 2.94 & 4.10 & 5.40 \\
& 4 & 1.56 & 2.94 & 4.28 & 5.80 \\
& 6 & 1.46 & 2.88 & 4.18 & 5.58 \\
& 8 & 1.52 & 2.84 & 4.14 & 6.06 \\
\hline
\end{tabular}

Moreover, in unvaried injection scenarios, the pressure drops of furan-based fuels are lower than diesel. Greater viscosities and densities accentuate the boundary layer on the orifice walls, causing a significant reduction in jet velocities. Thus, a reduction in pressure drop ensues.

However, it is worthy of attention that, under increasing backpressure and unvaried injection pressure, a different trend of pressure drops emerges from the comparison between the above fuels. With regard to diesel, increasing backpressure leads to a slightly decreasing trend of pressure drop, given the stronger contrasting action exerted by the pressurized vessel ambient on the fuel flow exiting the orifices. By contrast, both furan-based blends exhibit a fairly increasing trend of the pressure drops, which are always lower than diesel. Particularly, MF50 injections reveal a more accentuated increasing trend of the averaged pressure drops in comparison with MF30 shots.

The difference from diesel might be ascribable to the role exerted by the increasing backpressure on the needle motion. In fact, from a research activity (UK Patent Application No. 1819731.9) [20], it emerges that if on one hand increasing backpressure exerts a stronger barrier to the fuel flow, on the other hand, it is believed to boost the needle lift. This physical interpretation is based on the assumption that, at commencing injection event, the pressurized fuel momentum can be divided into two factors:

$M_{\mathrm{PF}}=M_{\mathrm{NP}}+M_{\mathrm{O}}$

with $M_{\mathrm{PF}}, M_{\mathrm{NP}}$, and $M_{\mathrm{O}}$ being the pressurized fuel momentum, the fuel momentum transferred onto the needle protrusion, and the fuel momentum towards the orifice inlet, respectively (Fig. 8).

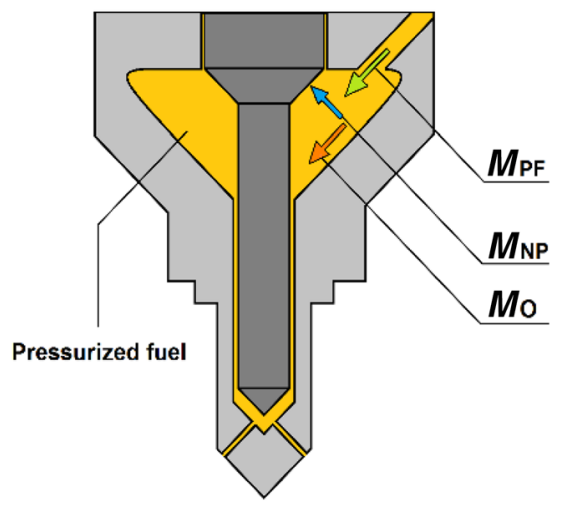

Fig. 8 Fuel momentum is subdivided into the fraction on the needle protrusion and the one toward the orifice

Accordingly, under constant injection pressure, the increasing backpressure exerts a stronger barrier to the fuel flow, mitigating the fuel momentum towards the orifices, for the benefit of momentum transferrable onto the needle. Thus, the needle experiences a larger acceleration.

By contrast, under constant injection pressure, low backpressure promotes the fuel flow towards the orifice inlet, reducing the fuel momentum transferrable onto the needle. Hence, a slower needle lift ensues.

Therefore, greater backpressures are believed to supply an additional acceleration to the needle. In this regard, in light of the observed trends of pressure drops, it might be presumed that the additional contribution of the backpressure to the needle lift is more appreciable for more viscous fuels. This is attributable to the more effective lubricant action outweighing the friction between the needle-control piston assembly and the internal part of the injector body. Thus, the needle moves upward more rapidly compared to lower fuel viscosity (but still larger than diesel one), advancing more immediately the opening of the cross-sectional area through which the fuel flows. In response to a more immediate needle lift, fuel experiences a more dramatic increase in kinetic energy, which causes a more abrupt reduction in pressure within the nozzle, according to Bernoulli's equation.

Different from diesel, MF30 and MF50 injections reveal a decreasing trend as a result of increasing backpressure under unvaried injection pressure. This might be attributable to the enhanced additional acceleration supplied by the greater backpressure on the needle, which experiences a faster upward motion. This results in a more abrupt opening of the cross-sectional area through which the fuel flows. Hence, fuel experiences a more dramatic increase in kinetic energy, implying a larger pressure drop, if compared to fuel provided with lower viscosity (but still larger than diesel one).

(2) The role of the backpressure in the rail pressure fluctuations 
This paragraph deals with the rail pressure fluctuations, focusing on the explanation of the retrieving time, the amplitudes of the rail pressure fluctuations, and the time interval between two consecutive pressure peaks acquired at each of the 16 injection configurations. Table 4 shows the retrieving times averaged over four injections and referring to Fig. 5.

It is observed that the diesel retrieving time is longer than MF30 one, which in turn is larger than MF50 in most cases. This is ascribable to the greater elasticity of furan-based blends in comparison with diesel. In this regard, a physical interpretation is provided in Sect. 4.

Moreover, as expected, an increase in injection pressure under unvaried backpressure tends to extend the time needed to retrieve the set rail pressure. Along with this observation, it can be seen that, under constant injection pressure for diesel injections, increasing backpressure implies a slightly decreasing trend of the retrieving time, except for the 70-8 MPa case. The discrepancy might be ascribed to the great instability of the rail pressure fluctuations induced by diesel injection. However, the trend from $70-2$ to $70-6 \mathrm{MPa}$ is strongly decreasing, in agreement with the rest of the cases.

By contrast, the MF30 and MF50 injection trends differ significantly from diesel. At higher viscosities than diesel, an increase in backpressure results in longer retrieving time. However, given the stronger opposing force of furan-based blends for counterbalancing the inertial effect stemming from the pressure perturbation, the MF30 and MF50 retrieving times are revealed to be shorter than diesel. The trend of furan-based blends retrieving times might be explained by the effect of greater viscosity combined with larger backpressure on the needle. As abovementioned, greater viscosity promotes the additional acceleration supplied by

Table 4 Retrieving time exhibited by tested fuels for each of set injection configurations

\begin{tabular}{llllll}
\hline Fuel & $\begin{array}{l}\text { Back pres- } \\
\text { sure (MPa) }\end{array}$ & \multicolumn{4}{l}{ Injection pressure } \\
\cline { 3 - 6 } & $40 \mathrm{MPa}$ & $70 \mathrm{MPa}$ & $100 \mathrm{MPa}$ & $140 \mathrm{MPa}$ \\
\hline \multicolumn{2}{l}{ Retrieving time (ms) } & & & & \\
Diesel & 2 & 257.5 & 322.5 & 330 & 425 \\
& 4 & 217.5 & 295 & 317.5 & 362.5 \\
& 6 & 235 & 270 & 302.5 & 347.5 \\
& 8 & 250 & 345 & 316.2 & 367.5 \\
MF30 & 2 & 212.5 & 255 & 282.5 & 352.5 \\
& 4 & 125 & 277.5 & 297.5 & 355 \\
& 6 & 170 & 265 & 252.5 & 337.5 \\
& 8 & 230 & 322.5 & 307 & 335 \\
MF50 & 2 & 117.5 & 205 & 215 & 307 \\
& 4 & 120 & 222.5 & 232.5 & 260 \\
& 6 & 135 & 205 & 235 & 325 \\
& 8 & 225 & 232.5 & 300 & 317.5 \\
\hline
\end{tabular}

backpressure on the needle. Thus, the needle experiences a more abrupt lift, giving rise to a more dramatic pressure drop. The ensuing rarefaction wave is provided with a larger magnitude. Once it reaches the rail, a longer time is required by the rail pressure to retrieve the value preceding the shot. Table 5 lists the amplitudes of pressure fluctuations following the injection shot and occurring over the retrieving time.

It is observable that diesel injection engenders larger amplitudes in comparison with MF30 and MF50 injections. Additionally, under unvaried backpressure, higher injection pressures lead to larger amplitudes. This might be owed to the greater pressure drop, which implies a larger magnitude of the rarefaction wave. As a result, wider amplitudes of pressure fluctuations ensue. On the other hand, under unvaried injection pressure, an increase in backpressure results in larger amplitudes in most cases. This might be owed to the boosting impact of the backpressure on the needle, faster lift of which is thought to engender a rarefaction wave provided with a larger magnitude. Thus, wider amplitudes of rail pressure fluctuation arise. Table 6 lists the averaged time between two consecutive peaks, indicating the pressure pulsations frequency.

It can be seen that diesel injection leads to a longer time interval between two consecutive pressure peaks in comparison with MF30 and MF50. Namely, the period of the diesel pressure fluctuations is significantly longer than the ones exhibited by furan-based blends. Furthermore, an increase in injection pressure extends the time intervals. Likewise, under unvaried injection pressure, greater backpressure leads to a longer time. Therefore, different from the pressure drops and retrieving time, the increase in fuel viscosity (but still larger than diesel) does not cause any increasing trend under unvaried injection pressure and increasing backpressure. The

Table 5 Amplitudes of the rail pressure fluctuations when altering the fuel, injection pressure, and backpressure

\begin{tabular}{llllll}
\hline Fuel & $\begin{array}{l}\text { Back pres- } \\
\text { sure (MPa) }\end{array}$ & \multicolumn{4}{l}{ Injection pressure } \\
\cline { 3 - 6 } & $40 \mathrm{MPa}$ & $70 \mathrm{MPa}$ & $100 \mathrm{MPa}$ & $140 \mathrm{MPa}$ \\
\hline Amplitude & of rail pressure fluctuations after & injection $(\mathrm{MPa})$ \\
Diesel & 2 & 2.25 & 3.10 & 3.75 & 3.50 \\
& 4 & 2.53 & 3.30 & 4.20 & 3.16 \\
& 6 & 2.40 & 3.40 & 4.0 & 3.75 \\
& 8 & 2.40 & 4.10 & 4.15 & 3.50 \\
MF30 & 2 & 0.40 & 0.70 & 1.10 & 1.15 \\
& 4 & 0.75 & 0.80 & 0.9 & 1.57 \\
& 6 & 0.75 & 0.95 & 1.33 & 1.90 \\
& 8 & 0.65 & 1.22 & 1.63 & 1.76 \\
MF50 & 2 & 0.28 & 0.75 & 0.5 & 0.70 \\
& 4 & 0.40 & 0.70 & 0.75 & 1.50 \\
& 6 & 0.70 & 0.80 & 0.85 & 1.53 \\
& 8 & 0.40 & 0.66 & 0.98 & 1.66 \\
\hline
\end{tabular}


Table 6 Duration of the time interval between two consecutive pressure peaks when altering the fuel, injection pressure, and backpressure

\begin{tabular}{llllll}
\hline Fuel & $\begin{array}{l}\text { Back pres- } \\
\text { sure (MPa) }\end{array}$ & \multicolumn{5}{l}{ Injection pressure } \\
\cline { 3 - 6 } & $40 \mathrm{MPa}$ & $70 \mathrm{MPa}$ & $100 \mathrm{MPa}$ & $140 \mathrm{MPa}$ \\
\hline \multicolumn{5}{l}{ Time between two consecutive pressure peaks } & $(\mathrm{ms})$ \\
Diesel & 2 & 100 & 100 & 95.5 & 75 \\
& 4 & 120 & 125 & 140 & 90 \\
& 6 & 100 & 105 & 100 & 90 \\
& 8 & 110 & 135 & 110 & 90 \\
MF30 & 2 & 40 & 40 & 50 & 76.6 \\
& 4 & 35 & 40 & 55 & 115 \\
& 6 & 45 & 35 & 50 & 76.6 \\
& 8 & 35 & 50 & 50 & 120 \\
MF50 & 2 & 25 & 25 & 30 & 45 \\
& 4 & 30 & 35 & 33.3 & 55 \\
& 6 & 30 & 35 & 30 & 60 \\
& 8 & 30 & 40 & 45 & 83.3 \\
\hline
\end{tabular}

combined impact of injection settings with fuel properties on rail pressure amplitude and frequency is explained in this section. However, the reasons underlying this trend reversal are worthy of further investigation.

\section{Discussion}

\subsection{Physical Principles Underlying the Experimental Results}

As already mentioned, the needle lift gives rise to an abrupt pressure drop, which generates a rarefaction wave traveling from the injector nozzle toward the rail. Similar to the dynamics effects attributable to the oncoming pressure wave from the delivery chamber, the propagation of the pressure wave is determined by fuel properties. It is well known that the speed $c$ of wave propagation across a liquid is approximately calculable via Eq. (1). Moreover, a larger bulk modulus enhances the speed of wave propagation. Assuming that liquid fuel is compounded with layers, the wave propagation is faster thanks to the accentuated reflection between the said layers, at the detriment of wave absorption. Such reflection generates inertial effects resulting in minimized oscillations amplitude. Therefore, it is presumable that a larger fluid stiffness is meaningful for the scarce propensity to absorb the wave energy. By contrast, the lower bulk modulus is meaningful for the greater fluid ability to absorb the wave energy, which is responsible for promoted amplitude oscillation. Hence, it is deductible that the lower bulk modulus accentuates the pressure fluctuations, underlying the spray nonuniformity, which affects adversely both the A/F ratio and the combustion efficiency. In the wake of these considerations, it may be inferred that the wave energy potentially transferrable to the fuel can be divided into two contributes. One contribution is associated with the wave reflection and is enhanced by higher bulk modulus, while the other one refers to the wave absorption, which contributes to pressure fluctuations amplitude. The magnitudes of the above factors are governed by the above fuel properties, such as bulk modulus. Additionally, while rail pressure is approaching the value preceding the start of the previous injection, the temporal interval between two consecutive pressure peaks is lower in the case of the larger bulk modulus. A further explanation may be provided by d'Alembert's equation, which describes the wave propagation through a fluid and is given by

$\frac{\delta^{2} p(x)}{\delta x^{2}}+\frac{\delta^{2} p(y)}{\delta y^{2}}+\frac{\delta^{2} p(z)}{\delta z^{2}}=\frac{1}{c^{2}} \frac{\delta^{2} p(t)}{\delta t^{2}}$

with $p, c$, and $t$ being the pressure acting on the fluid region involved by the wave propagation, wave speed, and time, respectively. In the present case, the d'Alembert's equation can be employed to describe the spatial distribution of the fluid pressure as a result of a wave perturbation traveling at velocity $c$. Keeping in mind that a larger bulk modulus boosts the speed of the pressure wave, it may be conjectured that the wave propagation generates less impactful effects on the pressure of the fluid region involved by the wave perturbation. Indeed, the pressure gradient is alleviated by increasing wave speed. Larger speed $c$ does not allow the wave energy to be sufficiently transferred to the fluid region involved by the wave propagation. Furthermore, the lower bulk modulus is deemed to imply a longer time required by fuel pressure to reach the value preceding the injection event, because of the above larger oscillation amplitude. Paying attention to the above curves and graphs, it may be argued that the large bulk modulus indicates the propensity of the fluid to recover the status preceding the advent of perturbation. Namely, the pressure of the liquid provided with the largest bulk modulus tends to approach more rapidly the value preceding the injection event. This can be further explained by the opponent force, which is determined by fuel properties and temperature, arisen in response to the deformation ensuing by the pressure wave. More specifically, according to Hooke's law applied to fluids, the opponent forces are determined by the fluid elasticity, which provides an estimation of the fluid propensity to retrieve the conditions preceding a perturbation. As such, these forces counterbalance the inertial effects stemming from the pressure perturbation, till the fluid status preceding the perturbation is restored. Additionally, compressibility provides an estimation of the change in volume induced by a variation in pressure, it might be presumed that greater compressibility 
implies a lower elasticity, which underlies the weaker opposing force. Accordingly, diesel rail pressure requires a longer time to retrieve the initial value. By contrast, the properties of furan, such as density and bulk modulus, lead to conjecture that the fluid particles are more closely positioned, making the furan-based blends less compressible than diesel. As such, opposing forces are stronger, shortening the retrieving time.

\subsection{Peculiarities of Furan-Blends Benefiting Rail Pressure Fluctuations}

In light of the considerations described in Sect. 3.2, it can be stated that the furan blends mitigate the margins of the unpredictability of the dynamic effects arising after the injection start. In fact, the pressure is revealed to retrieve more rapidly the value of rail pressure preceding the previous injection. As such, this minimizes the risk that the injector is energized when the above dynamical effects make injection pressure lower than the set value of the rail pressure, at the detriment of spray non-uniformity and the spatial distribution of the $\mathrm{A} / \mathrm{F}$ ratio. This represents a potential benefit for the optimization of the injection strategy, the setting of which is devoted to triggering the injector when injection pressure equals the set rail pressure or exceeds it, so as to enhance the spray penetration length and jet disintegration. Furthermore, modern injection systems enable the injection cycle to be split up into eight separate shots, the peculiarities of furan-based blends are further remarked by the consequential decrease in the dwell times between two consecutive shots. Indeed, the diminishing variation in rail pressure for the furan blends assures that rail pressure retrieves as rapidly as possible the value preceding the previous shot, so as to do not adverse the penetration length and radial propagation of the following shot.

\section{Conclusions}

The conclusions drawn from the experimental activity are summarized below:

1. As merely expected, higher viscosity and larger density are found to alleviate the pressure drop, which is accentuated by an increase in injection pressure. For instance, under injection pressure of $100 \mathrm{MPa}$ and backpressure of $6 \mathrm{MPa}$, the pressure drop of diesel, MF30, and MF50 is $6.28,4.66$, and $4.18 \mathrm{MPa}$, respectively.

2. Under unvaried injection pressure, greater backpressure softens the rail pressure drop of diesel. By contrast, MF30 and MF50 pressure drops tend to increase in response to greater backpressure. For instance, under injection pressure of $140 \mathrm{MPa}$ and increasing backpres- sure from 2 to $8 \mathrm{MPa}$, the diesel pressure drop decreases by $0.24 \mathrm{MPa}$. Conversely, MF30 and MF50 pressure drops increase by 0.12 and $0.66 \mathrm{MPa}$, respectively.

3. Under unvaried injection pressure and increasing backpressure, lower fuel viscosity (but still larger than diesel one) tends to alleviate the increase in rail pressure drop in most cases. For instance, under injection pressure of $40 \mathrm{MPa}$ and increasing backpressure from 2 to $8 \mathrm{MPa}$, MF30 and MF50 pressure drops increase by 0.26 and $0.38 \mathrm{MPa}$, respectively.

4. Under unvaried injection pressure and backpressures, lower fuel viscosity leads to longer retrieving time in most cases. For instance, under injection pressure of $100 \mathrm{MPa}$ and backpressure of $6 \mathrm{MPa}$, the retrieving time of MF50, MF30, and diesel is 235, 252.5, and $302.5 \mathrm{~ms}$, respectively.

5. Under unvaried injection pressure, the diesel retrieving time tends to be shortened in response to increasing backpressure in most cases. Conversely, greater backpressure tends to prolong MF30 and MF50 retrieving times. For instance, under injection pressure of $100 \mathrm{MPa}$ and increasing backpressure from 2 to $8 \mathrm{MPa}$, the diesel retrieving time decreases by $13.8 \mathrm{~ms}$. By contrast, MF30 and MF50 retrieving times increase by 24.5 and $85 \mathrm{~ms}$, respectively.

6. The amplitude of pressure pulsations is significantly accentuated in the case of fuel provided with low bulk modulus. Moreover, it has been observed that, under constant injection pressure and increasing backpressure, the amplitude of pressure fluctuations increases in most cases. For instance, under injection pressure of $100 \mathrm{MPa}$ and increasing backpressure from 2 to $8 \mathrm{MPa}$, the amplitude of pressure fluctuations for diesel, MF30, and MF50 increases by $0.40,0.53$, and $0.48 \mathrm{MPa}$, respectively.

7. Greater fuel bulk modulus promotes the frequency of the rail pressure pulsation at the detriment of the oscillation amplitude. Vice versa, fuel with lower bulk modulus is prone to pressure wave absorption, which leads to the wider amplitude of the pressure oscillation.

8. Over the stage during which the rail pressure approaches the value preceding the previous injection, the time interval between two consecutive pressure peaks is shorter in the case of a greater bulk modulus.

9. Under constant injection pressure and increasing backpressure, the time between two consecutive pressure peaks increases in most cases. For instance, under injection pressure of $70 \mathrm{MPa}$ and increasing backpressure from 2 to $8 \mathrm{MPa}$, the time interval for diesel, MF30, and MF50 increases by 35,10 , and $15 \mathrm{~ms}$, respectively.

The results emphasize the role of fuel viscosity and bulk modulus in pressure fluctuations underlying the uneven distribution of fuel flow in the injector orifices. This may spur 
the automotive industry to employ economical resources and man power for unlocking the potentialities of the furanbased blends, which provide a possible pathway for sustainable vehicular mobility.

Acknowledgement The present work is part of a 3-year research project sponsored by the Engineering and Physical Sciences Research Council (EPSRC) under Grant EP/N021746/1 and the National Natural Science Foundation of China (NSFC) under Grant 51.

\section{Compliance with Ethical Standards}

Conflict of interest On behalf of all the authors, the corresponding author states that there is no conflict of interest.

Open Access This article is licensed under a Creative Commons Attribution 4.0 International License, which permits use, sharing, adaptation, distribution and reproduction in any medium or format, as long as you give appropriate credit to the original author(s) and the source, provide a link to the Creative Commons licence, and indicate if changes were made. The images or other third party material in this article are included in the article's Creative Commons licence, unless indicated otherwise in a credit line to the material. If material is not included in the article's Creative Commons licence and your intended use is not permitted by statutory regulation or exceeds the permitted use, you will need to obtain permission directly from the copyright holder. To view a copy of this licence, visit http://creativecommons.org/licenses/by/4.0/.

\section{References}

1. Amirante, R., Coratella, C., Distaso, E., et al.: Optical device for measuring the injectors opening in common rail systems. Int. J. Automot. Technol. 18, 729-742 (2017)

2. Amirante, R., Catalano, L., Coratella, C.: A new optical sensor for the measurement of the displacement of the needle in a common rail injector. SAE Technical Paper 2013-24-0146 (2013)

3. Ubertini, S.: Injection pressure fluctuations model applied to a multidimensional code for diesel engines simulation. ASME. J. Eng. Gas Turbines Power. 128(3), 694-701 (2006)

4. Beierer, P., Huhtala, K., Lehto, E., Vilenius, M.: Study of the impact of system characteristics on pressure oscillations in a common rail diesel fuel injection system. SAE Technical Paper 200501-0910 (2005)

5. Catalano, L., Tondolo, V., Dadone, A.: Dynamic rise of pressure in the common-rail fuel injection system. SAE Technical Paper 2002-01-0210 (2002)
6. Catania, A.E., Ferrari, A., Manno, M., Spessa, E.: Experimental investigation of dynamics effects on multiple-injection common rail system performance. J. Eng. Gas Turbines Power. 130(3), 032806 (2008)

7. Pontoppidan, M., Ausiello, F., Bella, G., Ubertini, S.: Study of the impact on the spray shape stability and the combustion process of supply pressure fluctuations in CR-diesel injectors. SAE Technical Paper 2004-01-0023 (2004)

8. Li, P., Zhang, Y., Li, T., et al.: Elimination of fuel pressure fluctuation and multi-injection fuel mass deviation of high pressure common-rail fuel injection system. Chin. J. Mech. Eng. 28, 294$306(2015)$

9. Robinson, B., Bennett, J., Kilgore, J.: Bellows pressure pulsation damper. US Patent 5,845,621 (1998)

10. Wang, C., Xu, H., Lattimore, T.: Impacts of low-level 2-methylfuran content in gasoline on DISI engine combustion behavior and emissions. SAE Technical Paper 2013-01-1317 (2013)

11. Matthias, T., Martin, M., Stefan, P., et al.: Analysis of the impact of 2-methylfuran on mixture formation and combustion in a directinjection spark-ignition engine. Energy Fuels 25(12), 5549-5561 (2011)

12. Wang, C., Xu, H., Daniel, R., et al.: Combustion characteristics and emissions of 2-methylfuran compared to 2,5-dimethylfuran, gasoline and ethanol in a DISI engine. Fuel 103, 200-211 (2013)

13. Wang, Z., Ding, H., Wyszynski, M.L., et al.: Experimental study on diesel fuel injection characteristics under cold start conditions with single and split injection strategies. Fuel Process. Technol. 131(13), 213-222 (2015)

14. Esteban, B., Riba, J.R., Baquero, G., et al.: Temperature dependence of density and viscosity of vegetable oils. Biomass Bioenerg. 42, 164-171 (2012)

15. Jezak, S., Dzida, M., Zorebski, M.: High pressure physicochemical properties of 2-methylfuran and 2,5-dimethylfuran-second generation biofuels. Fuel 184, 334-343 (2016)

16. Shames, I.H.: Mechanics of Fluids. McGraw-Hill, New York (1982)

17. Zhmud, B.: Viscosity blending equations. Lube Mag. 121(93), 24-27 (2014)

18. Catania, A.E., Ferrari, A., Manno, M.: Development and application of a complete multijet common-rail injection-system mathematical model for hydrodynamic analysis and diagnostics. J. Eng. Gas Turbines Power. 130(6), 062809 (2008)

19. Wang, C., Sahu, A., Coratella, C., et al.: Spray characteristics of a gasoline-diesel blend (ULG75) using high-speed imaging techniques. Fuel 239, 677-692 (2019)

20. Coratella, C., Parry, L., Sahu, A., et al.: Optical sensor for the needle lift detection in the common rail injector. SAE Technical Paper 2019-24-0193 (2019) 